\title{
ON CREATIVE TEACHING OF ENGINEERING MECHANICS
}

\author{
HEINLOO, M[ati] \& AAN, A[are]
}

\begin{abstract}
This paper shows how to use the features of the Computer Package Mathcad for creative teaching of students in engineering mechanics by using its interactive worksheets and visualization the results of computations. As examples of visualization of the problems of engineering mechanics the simulations of motion of virtual four bar linkage, material point on the elliptical trajectory and large oscillations of a virtual double pendulum are considered.
\end{abstract}

Key words: creativity, interactivity, visualization, engineering mechanics, double pendulum

\section{INTRODUCTION}

The subjects of engineering mechanics contains a lot of formulas that are dead for engineering students before their visualization by numerical results of computations, graphs, their animations and simulations the motion of virtual objects.

Creative teaching method of engineering mechanics is based first of all on the interactive worksheets of computer packages. On these worksheets a student can change different parameters and initial conditions for studying virtual mechanical objects and make his own simulations of their motions or animations of the figures and examine these. The positive outcome of this method is that students have the possibility for more deep understanding of principles of mechanics. Negative outcome is the dependence of students from computers and special programs.

The purpose of this paper is to show how the Computer Package Mathcad (PTC Corporate, 2011) can be used for creative teaching of engineering mechanics.

\section{ON VISUALIZATION THE MOTION OF A FOURBAR}

Fig. 1 shows a frame of the video clip (Four bar linkage, 2011) that simulates the motion of a virtual four bar linkage (Norton, 1999) with a con-rod's curve.

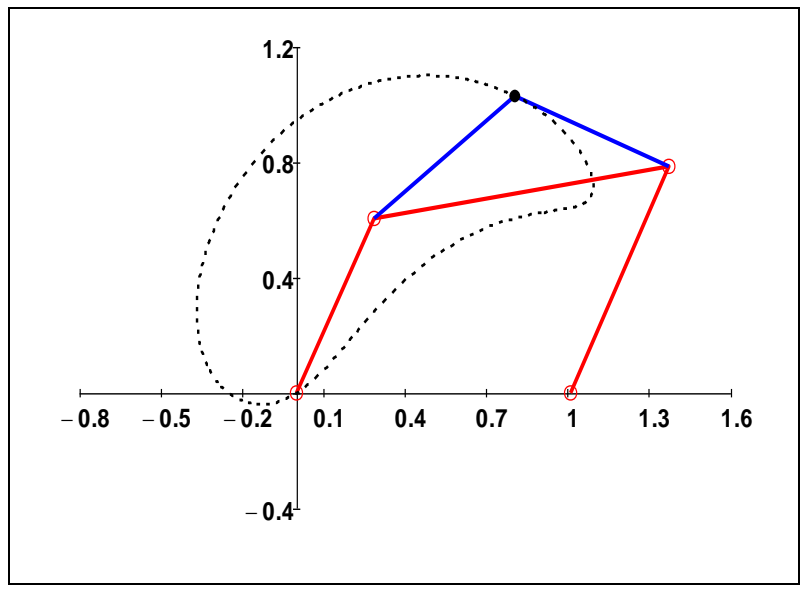

Fig. 1. A four bar linkage with a con-rod's curve

\section{ON VISUALIZATION THE MOTION OF A POINT}

Fig. 2 shows a frame from another video clip (Kinematics of a Point, 2011) that simulates the motion of a material point on the elliptical trajectory (Targ, 1988). This frame presents the circle of curvature at the point of trajectory, the centre of the curvature, the evolute that draws the centre of curvature and the arrows proportional to the real velocities and accelerations.

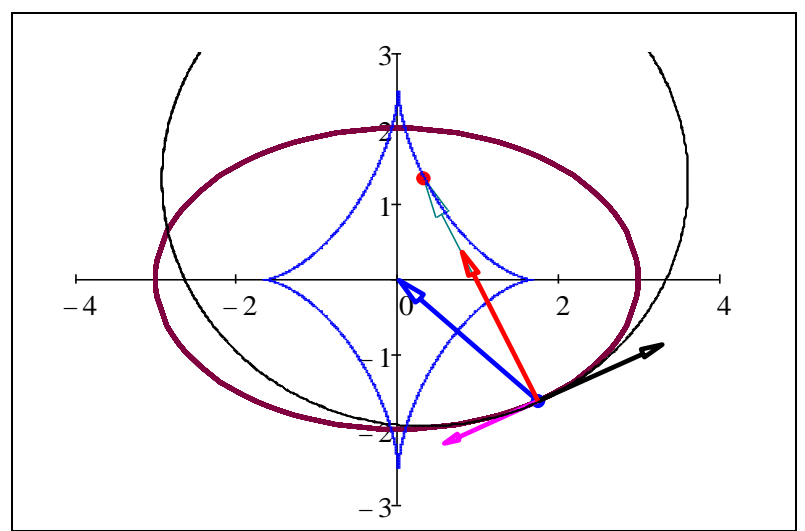

Fig. 2. The elliptical trajectory of the material point, its evolute, circle of curvature, vectors of velocity the accelerations of the moving point and the vector connecting moving point with the centre of curvature

\section{ON VISUALIZATION THE MOTION OF A DOUBLE PENDULUM}

Motion of a double pendulum can be modeled by the equations of Lagrange of second order or by the equations of Hamilton. It appears that large oscillations of a double pendulum are chaotic. A lot of results on study the double pendulums one can find on the different web sites, for example (Neumann, 2004; Wolfram demonstrations project). The real model of double pendulum is present, in video clip (High performance double pendulum, 2009). A double pendulum is presented in Fig. 3

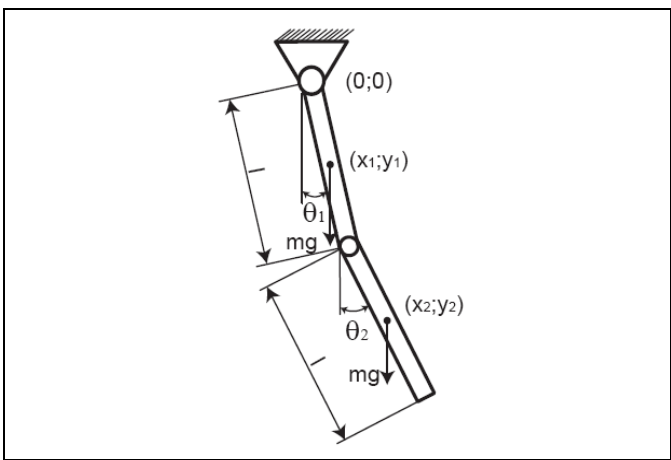

Fig. 3. A double pendulum 
The double pendulum in Fig. 1 consists two links, of equal lengths $l$ and weights $m g$, where $g$ is the acceleration. The mathematical model in the Hamilton form, describing large oscillations of the double pendulum is (Wikipedia, 2011)

$$
\begin{aligned}
& \dot{\theta}_{1}=\frac{6}{m l^{2}} \frac{2 p_{\theta_{1}}-3 p_{\theta_{2}} \cos \left(\theta_{1}-\theta_{2}\right)}{16-9 \cos \left(\theta_{1}-\theta_{2}\right)^{2}}, \\
& \dot{\theta}_{2}=\frac{6}{m l^{2}} \frac{8 p_{\theta_{2}}-3 p_{\theta_{1}} \cos \left(\theta_{1}-\theta_{2}\right)}{16-9 \cos \left(\theta_{1}-\theta_{2}\right)^{2}}, \\
& \dot{p}_{\theta_{1}}=-\frac{1}{2} m l^{2}\left(\dot{\theta}_{1} \dot{\theta}_{2} \sin \left(\theta_{1}-\theta_{2}\right)+3 \frac{g}{l} \sin \left(\theta_{1}\right)\right), \\
& \dot{p}_{\theta_{2}}=-\frac{1}{2} m l^{2}\left(-\dot{\theta}_{1} \dot{\theta}_{2} \sin \left(\theta_{1}-\theta_{2}\right)+\frac{g}{l} \sin \left(\theta_{2}\right)\right),
\end{aligned}
$$

where $\theta_{1}$ and $\theta_{2}$ are generalized co-ordinates (Wikipedia, 2011), or inclination angles, of the double pendulum (Fig. 3) and

$$
\begin{aligned}
& p_{\theta_{1}}=\frac{1}{6} m l^{2}\left(8 \dot{\theta}_{1}+3 \dot{\theta}_{2} \cos \left(\theta_{1}-\theta_{2}\right)\right), \\
& p_{\theta_{2}}=\frac{1}{6} m l^{2}\left(2 \dot{\theta}_{2}+3 \dot{\theta}_{1} \cos \left(\theta_{1}-\theta_{2}\right)\right),
\end{aligned}
$$

are the generalized impulses (Lepik \& Roots 1971) of the double pendulum.

Let us assume that the special solution of the equations (1) satisfies the following initial conditions:

$$
\theta_{1}(0)=\theta_{10}, \theta_{2}(0)=\theta_{20}, p_{\theta_{1}}(0)=p_{\theta_{10}}, p_{\theta_{2}}(0)=p_{\theta_{20}},
$$

where $\theta_{10}, \theta_{10}, p_{\theta_{10}}, p_{\theta_{20}}$ are constants. Let us consider the initial position of the double pendulum, shown in Fig. 4 and assume that the motion of double pendulum begins without initial angular velocities.

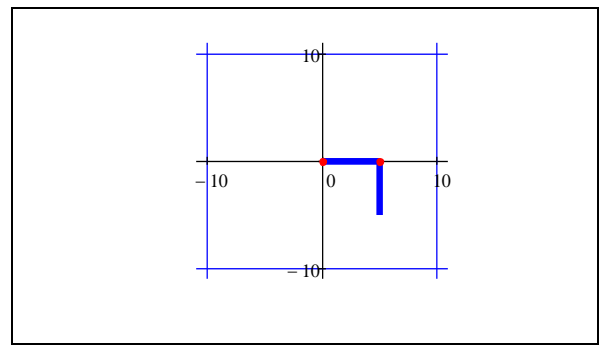

Fig. 4. First initial position of the double pendulum

This assumption means that in the initial conditions (2) the constants must have the following values

$$
\theta_{10}=\frac{\pi}{2}, \theta_{20}=0, p_{\theta_{10}}=0, p_{\theta_{20}}=0 .
$$

The system of equations (1) under conditions (2), (3) (Fig. 4) was solved on the worksheet of the Computer Package Mathcad by using the numerical algorithm of Runge-Kutta.

The computations were made for $l=5, m=1, g=9.807$ in the case of initial values (3) and visualized in Figs. 5 and 6.

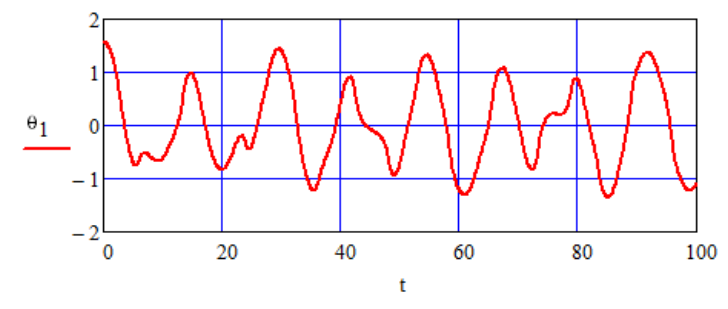

Fig. 5. The dependence of inclination angle $\theta_{1}$ on time in the case of initial values (3) (Fig. 4)

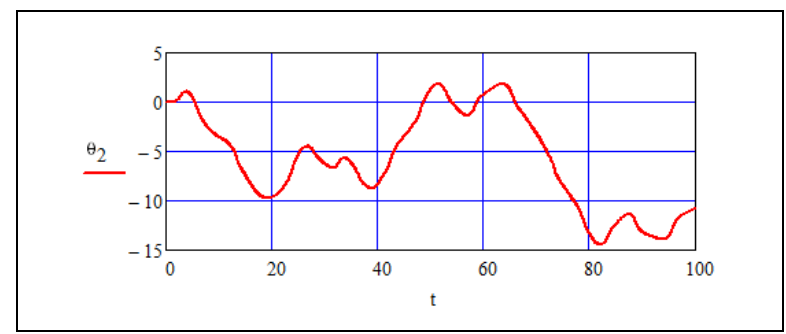

Fig. 6. The dependence of inclination angle $\theta_{2}$ on time in the case of initial values (3) (Fig. 4)

The diagrams in Figs 5-6 and the video clip (Physical double pendulum, 2011) show the chaotic motion of double pendulum. Fig. 7 shows several frames from this video clip

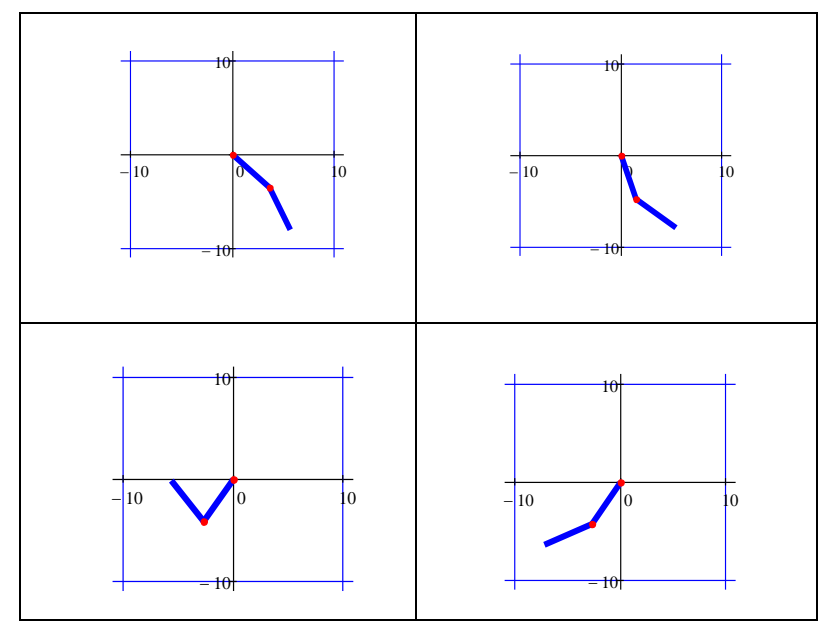

Fig. 7. Frames from composed video clip from initial position in Fig. 4

\section{CONCLUSIONS}

The enrichment of engineering mechanics by the features of the information technology increases the efficiency of studying different subjects of engineering mechanics and makes teaching process more interesting both for students and teachers.

\section{REFERENCES}

Norton, R. L. (1999). Design of Machinery, An Introduction to the Synthesis and Analysis of Mechanisms and Machines, Second edition, McGraw-Hill, ISBN, Boston

Neumann, E. (2004) Double pendulum, http://www.myphysicslab.com/dbl_pendulum.html, Accessed on: 2011-02-13

PTC Corporate. (2011). Mathcad. http://www.ptc.com/products/mathcad/, Accessed on: 2011$01-13$

Targ, S. (1988). Theoretical Mechanics, Moscow

Lepik, Ü; Roots, L. (1971). Theoretical Mechanics, Tallinn

*** (2011) Wikipedia. Double pendulum, http://en.wikipedia.org/wiki/Double_pendulum, Accessed on: 2011-03-03

*** Wolfram demonstrations project. Double pendulum, http://demonstrations.wolfram.com/DoublePendulum/, Accessed on: 2011-02-13

*** (2011) Kinematics of a Point, http://www.youtube.com/watch? $\mathrm{v}=\mathrm{zFbVcd} 4 \mathrm{GOJw}, A c$ cessed on: 2011-01-17

*** (2011) Four bar linkage, http://www.youtube.com/watch?v=GgEra0JPoDU, Accessed on: 2011-01-15

*** (2011) Physical double pendulum, http://www.youtube.com/watch?v=BFIJTAzYdvo, $A c$ cessed on: 2011-03-13

*** (2009) High performance double pendulum, http://www.youtube.com/watch?v=z3W5aw-VKKA, Accessed on: 2011-02-13 